Rohr in das darunter befindliche Kölbchen abfliesst. Ferner ist die Form des Gefässes (A) durch eine abgeplattete Ausbauchung der Wandung derartig gestaltet, dass der Apparat zur Vornahme von Trockensubstanz-Bestimmungen in nicht festen Substanzen wagerecht gelegt werden kann. Von Vortheil ist, dass die Abwägung des Untersuchungsmateriales direkt in dem etwa $20 \mathrm{~g}$ schweren Apparat erfolgen kann, und dass die Mengen der extrahirten Substanzen durch den Gewichtsverlust sich schnell festsetzen lassen. Angefertigt wird der Apparat bei Julius Elsner in Goldlauter i. Th. und bei Franz Hugershoff in Leipzig.

P. Buttenberg.

\title{
Die Eiweisskörper und deren Beurtheilung vom ernährungsphysiologischen Standpunkte.
}

Von Dr. A. Jolles in Wien.

Das Studium der Eiweisskörper war früher mit grossen Schwierigkeiten verknüpft, weil es nicht möglich war, genügend reines Untersuchungsmaterial zu beschaffen. Durch die Möglichkeit der Krystallisation lassen sich jetzt diese Körper zum grossen Theil derartig rein darstellen, dass man nicht mehr befürchten muss, mit Gemischen zu arbeiten.

Die chemische Untersuchung der Eiweisskörper bezweckt vornehmlich deren Konstitution kennen zu lernen. Die Elementaranalyse allein genügt dazu nicht. Man versucht daher, den Begriff der Eiweisskörper durch Gruppenreaktionen schärfer zu fassen und durch Ausmittelung der Spaltungsprodukte des Eiweisses bei den verschiedenen Reaktionen den erwünschten Aufschluss zu erhalten. Mit der Ueberführung des Eiweisses in einfachere Atomkomplexe befassen sich besonders die Arbeiten von Kossel. Durch kochende Säuren gelang es ihm, aus Eiweisskörpern aliphatische stickstoffhaltige Körper aus der Reihe der Amidosäuren und deren Abkömmlinge, wie Arginin, Histidin und Lysin darzustellen, die sämmtlich in Molekül 6 Atome Kohlenstoff enthalten und daher von Kossel Hexonbasen genannt wurden. Da nun die Protamine, die einfachsten bisher bekannten Eiweisskörper, bei der Spaltung nur Hexonbasen liefern, so nimmt Kossel an, dass in Eiweisskörpern die Hexonbasen den Kern bilden, an welchen andere Reste angelagert sind. Einen genauen Aufschluss über die Konstitution der Eiweisskörper gaben die bisherigen Arbeiten nicht. Jolles hat die Spaltung des Eiweisses mit Permanganat in sauerer Lösung ausgeführt und dabei gefunden, dass bestimmte Körper wie Harnstoff, Mono- und Diamidosäuren nicht wie bei der alkalischen Spaltung weiter zerlegt werden. Auf diesem Wege gelingt es, gerade wie im Organismus, das Eiweiss bis zum Harnstoff abzubauen. Dabei geht der grösste Theil des Stickstoffes in Harnstoff über, der Rest dagegen in durch Phosphorwolframsäure fällbare Verbindungen. Weiter zeigt Jolles, dass der Harnstoff aus der Gruppe $\mathrm{CONH}_{2}$ bezw. CONH hervorgeht und führt als Beispiele die Ureïde, die Purinbasen, Hippursäure, Asparagin, Laktamid, Succinamid, Benzoylasparaginsäure an. Bei Untersuchung von Eiweisskörpern gaben dieselben über $45 \%$ zuweilen sogar bis $90 \%$ ihres Stickstoffes in Form von Harnstoff ab. Es müssen daher in dem Eiweisskörper viele harnstoffbildende Gruppen in wechselnder Menge enthalten sein. Der Stickstoff des Eiweisses, welcher nicht als Harnstoff abgeschieden wird, giebt beim Abbau hauptsächlich die Kossel'schen Hexonbasen. Auf Grund theoretischer Erwägungen schreibt Jolles denjenigen Gruppen, welche Harnstoff liefern, am meisten die ernährende Wirkung zu und hat den dazu nöthigen Beweis auf experimentellem Wege erbracht. 
Bei Fütterungsversuchen mit zwei Eiweisskörpern (Kaseïn und Fibrin), die sich bei der Oxydation ganz verschieden verhalten, konnte nachgewiesen werden, dass die harnstoffbildenden Gruppen wichtig für die Ernährung sind. Den Gruppen, welche den Stickstoff nicht in Form von Harnstoff liefern, kommt eine bedeutend geringere Ausnutzbarkeit zu. Dabei fand sich ferner, dass die Hexonbasen nicht in den Urin sondern in die Fäces übergehen. Da nun je nach der Bindungsform der Stickstoff bei der Ernährung eine verschiedene Rolle spielt, so lässt sich durch die übliche Analyse eines Eiweisskörpers dessen wahrer Nährwerth nicht feststellen; derselbe Uebelstand zeigt sich, wenn man durch die kalorimetrische Messung des Verbrennungswerthes eines Eiweisskörpers den Energiebetrag, welcher beim Verbrauch dieses Nährstoffes im Organismus auftritt, wiedergeben will.

P. Buttenberg.

\title{
Die Beurtheilung der Süd- tud Süssweine, insbesondere der Ungarweine unter Berücksichtigung der neuen gesetzlichen Bestimmungen.
}

\author{
Von Dr. Bein in Berlin.
}

Mit Bezug auf seinen im Jahre 1896 auf der Naturforscher-Versammlung gehaltenen Vortrag führt der Vortragende aus, dass nach $\S 2^{1}$ und $\S 8$ des mit dem 1 . Oktober 1901 in Kraft tretenden neuen Weingesetzes vom 24. Mai 1901 für Dessertweine (Süd- und Süssweine) eine Beschränkung des Alkoholzusatzes bezw. eine Grenze für den Gehalt an Kaliumsulfat nicht besteht.

Nur sofern die Süd- und Süssweine als Medicinalweine feilgehalten werden, müssen dieselben den im deutschen Arzneibuch Ausgabe IV gestellten Anforderungen bezüglich Alkohol und Kaliumsulfatgehalt entsprechen.

Für Ungarweine sind die Landesgesetze weit strengere, da diéselben nach der ungarischen Gesetzgebung weder mit Alkohol versetzt werden dürfen noch durch die übliche Kellerbehandlung eine Weinvermehrung stattfinden darf.

Nach Vortragendem enthalten nun gerade die besseren Ungarweine weniger Alkohol und mehr Extrakt als nach dem deutschen Arzneibuch für diese gefordert wird, so dass dieselben in Folge dessen nur als Süss- oder Qualitätsweine nicht aber als Südweine in den Apotheken geführt werden dürfen. [Vergl. hierzu diese Zeitschrift 1901, 4, 1144 - Red.]

Für Ungarweine des sogen. Tokayer-Gebietes ist eine Zuckerung, wie sie für Weine des deutschen Weinbaugebietes zulässig ist, selbst unter Deklaration nach der ungarischen Gesetzgebung verboten. Die Süsse der Medicinal-Ungarweine darf nur durch Verwendung von Trockenbeeren bedingt sein, und für die anderen süssen Ungarweine ist eine Zuckerung nur dann gestattet, wenn hierdurch eine Verbesserung der Weine erzielt wird, ohne dass dabei eine wesentliche Vermehrung derselben stattfindet

Das neue deutsche Weingesetz gestattet dagegen, wie das frühere, die Zuckerung der Weine, sofern dieselben hierdurch nicht die Durchschnittszusammensetzung der Weine des betreffenden Weinbaugebietes unterschreiten.

Nach Vortragendem sind vermittelst getrockneter Früchte in Deutschland hergestellte künstliche Dessertweine entsprechend den gesetzlichen Bestimmungen als künstlich hergestellte zu bezeichnen und ist daher der Betrieb vorher bei der Behörde anzumelden.

K.Lendrich. 